\title{
Denosumab for the treatment of HIV-associated osteoporosis with fractures in a premenopausal woman
}

\author{
E. Marasco ${ }^{1}$, M. Mussa ${ }^{2}$, F. Motta ${ }^{1}$, F. Bobbio-Pallavicini' ${ }^{1}$, R. Maserati ${ }^{2}$, \\ C. Montecucco ${ }^{1}$, L. Bogliolo ${ }^{1}$ \\ 'Unit of Rheumatology, Policlinico San Matteo IRCCS Foundation, University of Pavia, Italy; \\ 2Infectious Disease Department, Policlinico San Matteo IRCCS Foundation, Pavia, Italy
}

\section{SUMMARY}

The prevalence of osteoporosis is about three times greater in people living with HIV than in the general population. Bisphosphonates are the only class of antiresorptive drugs which have proved to be safe and effective in HIV patients. However, bisphosphonates are not recommended in women of childbearing age due to an increased rate of associated neonatal complications. To the best of our knowledge no reports on the use of denosumab in HIV-infected individuals have been published so far. We describe a 38 year-old woman with HIV, osteoporosis and vertebral fractures treated with denosumab, a monoclonal antibody targeting RANKL. After four years of treatment, bone mineral density improved, no new fractures occurred, and neither HIV reactivation nor opportunistic infections were observed. We show that denosumab could be a safe and effective approach for osteoporosis in patients with HIV and could be considered in women of childbearing age.

Key words: Osteoporosis, HIV, denosumab.

Reumatismo, 2021; 73 (1): 54-58

\section{INTRODUCTION}

The prevalence of osteoporosis (OP) in patients with human immunodeficiency virus (HIV) infection can be as high as $21 \%$, about three times greater than in the general population (1). Several factors play a role in the development of HIV-associated OP: viral-induced osteoclastic activity, altered balance in the ligand of receptor activator for nuclear $\kappa \mathrm{B}$ (RANK)/Osteoprotegerin (OPG) axis, chronic inflammation and antiretroviral therapy (ART) (2). ART initiation elicits the activation of T-cell reconstitution producing RANKL, enhancing bone resorption and bone mineral density (BMD) loss (2).

Denosumab (Dmab) is a monoclonal antibody with antiresorptive properties directed against the RANKL; it prevents the binding of RANK to RANKL, inhibiting the maturation of osteoclasts and impairing their survival (3). Dmab is currently approved for the treatment of OP in men- opausal women and men with increased fracture risk (3). Dmab did not increase the risk of developing infections (3); moreover, a study demonstrated its safety also in patients with rheumatoid arthritis treated with immunosuppressants (4).

To the best of our knowledge no controlled studies on the use of Dmab in HIV-infected individuals have been published so far (5, 6 ). We report the case of a woman of fertile age with HIV infection and OP with fractures treated with Dmab.

\section{CASE REPORT}

A 38 year-old woman was referred to our clinic in 2014 for fractures of the $9^{\text {th }}$ and $11^{\text {th }}$ thoracic vertebrae and OP. She reported sudden onset of non-traumatic back pain. An X-ray of the spine showed two vertebral fractures. No history of fragility fractures was present. Following the discovery of vertebral fractures, a bone densitometry (Dual-energy X-ray absorptiom- 
etry or DXA) performed at another center revealed a Z-score at the lumbar spine (L1-L4) of -2.7 SD (T score -3.1 SD). No previous DXA scan had been performed before for comparison. In 2007, she was diagnosed HIV positive with a HIV-RNA of 199,088 copies/mL and a nadir of CD4 ${ }^{+} \mathrm{T}$ cells of 118 cells $/ \mu \mathrm{L}$. ART with tenofovirdisoproxil-fumarate (TDF)/emtricitabine and atazanavir/ritonavir was initiated. Three months later, she was switched to a TDF-free regimen for renal toxicity. In $\mathrm{Au}-$ gust 2018, she was started on rilpivirine/ tenofovir-alefenamide/emtricitabine. Since 2010, the viral load had been constantly suppressed and $\mathrm{CD} 4^{+} \mathrm{T}$ cell count remained above 400 cells/ $\mu \mathrm{L}$. Examination of lungs, heart and abdomen was normal. Laboratory workup revealed normal cell blood counts, renal and liver functions; normal levels of calcium, phosphate, $25 \mathrm{OH}$-vitamin $\mathrm{D}$, alkaline phosphatase, $\mathrm{PTH}$, thyroid hormones, urinary excretion of calcium and phosphate.

The patient was eligible to start antiresorptive therapy considering her two vertebral fractures, her general good health and the successful treatment of HIV with ART. Bisphosphonates (BPs) have shown to be safe and effective in people with HIV; however, since these drugs are associated with an increased rate of neonatal complications and persist in the bone matrix for years even after therapy discontinuation, fetal exposure during pregnancy is a concern and their use is not recommended, if a pregnancy is planned in the following 12 months (7). Fetal exposure to BPs has been associated with shortened gestational age, low neonatal birth weight and transient hypocalcaemia of the newborns (8). Dmab is effective in post-menopausal OP with fractures; however, there is no data on its safety in people with HIV. We discussed with the patient the safety and efficacy of the different antiresorptive therapies. The patient decided to initiate Dmab after considering the pros and cons of the two different treatment approaches, taking into account her desire to become pregnant in the future. The drug was prescribed, with informed consent, offlabel, as the patient was still of childbear-

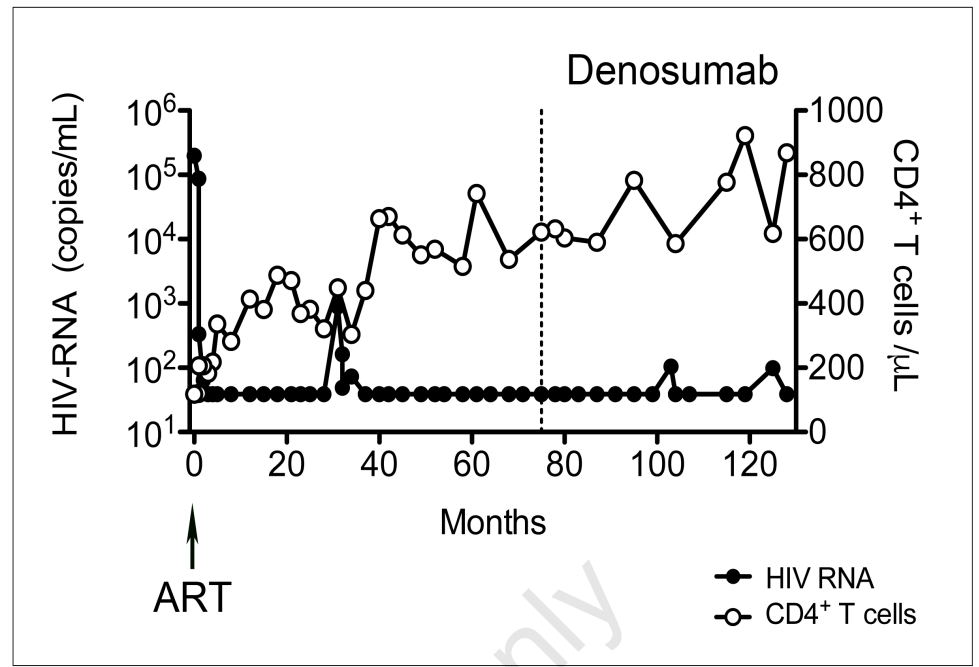

Figure 1 - HIV-RNA viral load and CD4+T cell count from diagnosis (month 0) and after treatment with denosumab; the arrow indicates initiation of ART; the dashed line indicates initiation of denosumab treatment.

ing age. Dmab $60 \mathrm{mg}$ was administered subcutaneously every six months. A high calcium diet and vitamin D supplementation were also recommended. The patient was advised about effective contraception during treatment with Dmab. During the 53 month follow-up, no new fractures appeared. At a DXA control performed at our centre after 18 months, spinal (L1-L4) Zscore was -2.5 SD (T score -2.6 SD) (BMD $\left.0.765 \mathrm{~g} / \mathrm{cm}^{2}\right)$ and femoral neck Z-score was -2.9 SD (T score -3.1 SD) (BMD $0.509 \mathrm{~g} /$ $\mathrm{cm}^{2}$ ); after 40 months, spinal (L1-L4) Zscore further improved to $-2.2 \mathrm{SD}$ ( $\mathrm{T}$ score $-2.4 \mathrm{SD}$ ) (BMD $0.784 \mathrm{~g} / \mathrm{cm}^{2}$ ), and femoral neck Z-score improved to -2.6 SD (T score $-2.8 \mathrm{SD}$ ) (BMD $0.538 \mathrm{~g} / \mathrm{cm}^{2}$ ). The HIV viremia remained suppressed and $\mathrm{CD} 4^{+} \mathrm{T}$ cell count remained higher than 600 cells/ $\mu \mathrm{L}$ throughout the follow-up period (Figure 1). Dmab was well tolerated, with no side effects. Particularly, no episodes of hypocalcemia and no infectious complications were reported.

\section{DISCUSSION AND CONCLUSIONS}

OP is a systemic skeletal disease characterized by low BMD and deterioration of the bone microarchitecture, resulting in a 
loss of bone strength, increased bone fragility, and an increased risk of fractures. In the past two decades, the life expectancy of people living with HIV infection has increased significantly, and OP and fragility fractures have emerged as significant comorbidities (9). For this reason, it is now recommended to assess and monitor BMD in people with HIV carrying other risk factors for developing OP and fragility fractures (FF). Treatment with vitamin D and calcium supplementation and antiresorptive therapy with BPs are recommended in patients with HIV and either OP or a high risk of FF (9).

Mechanisms of bone loss in the setting of HIV infection are multifactorial and include viral, host, and immune factors, as well as direct and indirect effects of ART. First, HIV viral proteins promote osteoclast activity and induce apoptosis of osteoblasts, uncoupling bone resorption and bone formation. Moreover, HIV induces the production of pro-inflammatory cytokines that activate osteoclasts and bone resorption (2). B cells are also involved in bone loss as HIV infection increases the production of RANKL by B cells (2). Initiation of ART, restoring $\mathrm{T}$ cell numbers, is associated with a paradoxical reduction in BMD, which is in part independent of the type of ART regimen. Mouse models of HIV infection helped to understand that $\mathrm{T}$ cell reconstitution elicits the production of RANKL and TNF by T cells. The proinflammatory milieu associated with immune reconstitution promotes BMD loss. In addition, several antiretrovirals have been associated with BMD loss (10). The drugs that appeared to be mostly associated with BMD loss are TDF and protease inhibitors (PIs). TDF alters tubular function, causes hypophosphatemia - consequently increasing PTH levels - and a reduction in the levels of 1,25-dihydroxyvitamin $\mathrm{D}$, thus, promoting bone resorption (11). Newer antiretrovirals are associated with less bone loss than PIs and TDF (2). All in all, factors related both to HIV per se and to its treatment are implicated in the development of HIV-associated OP (2) and an imbalance in the RANKL/OPG axis ap- pears to play a crucial role in this process. The use of BPs has been shown to be safe and effective in HIV patients. Emerging data indicate that BMD loss following ART initiation can be mitigated by prophylaxis with BPs, resulting in an improvement in BMD and a significant reduction in fracture risk (9). BPs exert their antiresorptive effects accumulating in the bone matrix where osteoclasts are actively promoting bone resorption. They can persist in the bone matrix for years (up to 10 years for alendronate), even after therapy is discontinued. The long half-life of BPs poses a problem in pre-menopausal women, since there might be a risk of fetal exposure in case of pregnancy. Fetal exposure to BPs has been associated with shortened gestational age, low neonatal birth weight and transient hypocalcaemia of the newborns (8).

Dmab, a fully human recombinant monoclonal antibody that targets RANKL, has shown to increase BMD and prevent fractures in both women and men $(3,12)$. The safety of Dmab has been extensively studied in large phase III trials and their extension trials, showing no differences in the overall incidence of infections and serious adverse events of all types $(3,13)$. Moreover, Dmab has proven effective and safe in patients with autoimmune diseases treated with biologics (4). Biologics are safe to use in HIV infected people, if viremia is suppressed and $\mathrm{CD} 4^{+} \mathrm{T}$ cell count is higher than 200/uL (14). The same principles can be applied to the use of Dmab in this group of patients. The blockade of the RANK/ RANKL axis in patients with HIV is a promising target, as this has shown to play a crucial role in the development of HIVassociated OP on multiple experimental settings and in in vivo studies. Unlike BPs, Dmab targets osteoclasts at a more immature stage, with an on-off action, reversible upon treatment discontinuation. The physiological bone turnover is completely restored after 6 months of Dmab discontinuation. The on-off mode of action of Dmab poses an advantage over BPs for its use in young women: upon drug discontinuation, Dmab can be cleared from the organism, 
reducing significantly the risk of fetal exposure, if timing for conception is respected. It is important to note that in women with postmenopausal OP, Dmab discontinuation was associated with a decrease in BMD and an increased risk of fracture (15), posing a possible contraindication for its use in young women. However, we do not know if the same applies to women of childbearing age, as estrogens may have a protective effect. In women with breast cancer treated with aromatase inhibitors and Dmab, the discontinuation of Dmab was associated with an increased risk of fractures, when aromatase inhibitors were stopped six months after Dmab (16).

Another important factor to consider is that Dmab is not contraindicated in patients with OP associated with chronic kidney damage (CKD). Dmab, unlike BPs, can be employed also in patients on hemodialysis (17). It is worth mentioning that renal impairment and haemodialysis are associated with an increased risk of hypocalcaemia, especially if proper calcium and vitamin D supplementation is not prescribed, and patients should be monitored closely for the aforementioned side effect. Moreover, Dmab and all antiresorptive drugs cannot be used in patients with adynamic bone disease. The potential use in patients with CKD constitutes another advantage for Dmab over BPs, as people living with HIV have a higher prevalence of CKD.

Our case report shows that Dmab could be safe and effective in people living with HIV and with osteoporotic fractures. Neither viral reactivation nor major infective events were observed in our patient during Dmab treatment. This work provides the ground to implement clinical trials to investigate the safety and efficacy of Dmab as antiresoptive agent in HIV-associated $\mathrm{OP}$, even in young fertile women.

\section{Conflict of interests}

The authors declare no conflict of interests.

\section{REFERENCES}

1. Brown TT, Qaqish RB. Antiretroviral therapy and the prevalence of osteopenia and osteopo- rosis: a meta-analytic review. AIDS (London, England). 2006; 20: 2165-74.

2. Moran CA, Weitzmann MN, Ofotokun I. Bone Loss in HIV Infection. Curr Treat Options Infect Dis. 2017; 9: 52-67.

3. Cummings SR, San Martin J, McClung MR, et al. Denosumab for prevention of fractures in postmenopausal women with osteoporosis. N Engl J Med. 2009; 361: 756-65.

4. Lau AN, Wong-Pack M, Rodjanapiches R, et al. Occurrence of serious infection in patients with rheumatoid arthritis treated with biologics and denosumab observed in a clinical setting. J Rheumatol. 2018; 45: 170-6.

5. Premaor MO, Compston JE. The hidden burden of fractures in people living with HIV. JBMR Plus. 2018; 2: 247-56.

6. de la Morena IVS, Paz Solarte JA, Bedoya D, Galindo MJ. Is denosumab safe in HIV patients? EULAR 2019. Ann Rheum Dis. 2019; 78: A1884.

7. Langdahl BL. Osteoporosis in premenopausal women. Curr Opin Rheumatol. 2017; 29: 410-5.

8. Stathopoulos IP, Liakou CG, Katsalira A, et al. The use of bisphosphonates in women prior to or during pregnancy and lactation. Hormones (Athens, Greece). 2011; 10: 280-91.

9. McComsey GA, Tebas P, Shane E, et al. Bone disease in HIV infection: a practical review and recommendations for HIV care providers. Clin Infect Dis. 2010; 51: 937-46.

10. Heath KV, Montaner JS, Bondy G, et al. Emerging drug toxicities of highly active antiretroviral therapy for human immunodeficiency virus (HIV) infection. Curr Drug Targets. 2003; 4: 13-22.

11. Masia M, Padilla S, Robledano C, et al. Early changes in parathyroid hormone concentrations in HIV-infected patients initiating antiretroviral therapy with tenofovir. AIDS Res Hum Retrovir. 2012; 28: 242-6.

12. Orwoll E, Teglbjaerg CS, Langdahl BL, et al. A randomized, placebo-controlled study of the effects of denosumab for the treatment of men with low bone mineral density. J Clin Endocrinol Metab. 2012; 97: 3161-9.

13. Papapoulos S, Lippuner K, Roux C, et al. The effect of 8 or 5 years of denosumab treatment in postmenopausal women with osteoporosis: results from the FREEDOM Extension study. Osteoporos Int. 2015; 26: 2773-83.

14. Louthrenoo W. Treatment considerations in patients with concomitant viral infection and autoimmune rheumatic diseases. Best Pract Res Clin Rheumatol. 2015; 29: 319-42.

15. Cummings SR, Ferrari S, Eastell R, et al. Vertebral fractures after discontinuation of denosumab: a post hoc analysis of the randomized placebo-controlled FREEDOM Trial and Its Extension. J Bone Mineral Res. 2018; 33: 190-8. 
16. Pfeiler GS, Egle D, Greil R, et al. Fracture risk after stopping adjuvant denosumab in hormone receptor positive breast cancer patients on aromatase inhibitor therapy - an analysis of 3,425 postmenopausal patients in the phase III ABCSG-18 trial. ASBMR 2018 Annual Meet- ing, 2018: Oral Presentations, Presentation Number: LB-1167.

17. Kunizawa K, Hiramatsu R, Hoshino J, et al. Denosumab for dialysis patients with osteoporosis: A cohort study. Sci Rep. 2020; 10: 2496. 